\title{
Role of CDK8 and B-catenin in colorectal adenocarcinoma
}

\author{
JONG-OG SEO ${ }^{1}$, SONG IY HAN ${ }^{2}$ and SUNG-CHUL LIM ${ }^{1,2}$ \\ ${ }^{1}$ Department of Pathology and ${ }^{2}$ Research Center for Resistant Cells, Chosun University \\ School of Medicine, 588, Seosuk-dong, Dong-gu, Gwangju, Republic of Korea
}

Received March 5, 2010; Accepted April 29, 2010

DOI: 10.3892/or_00000858

\begin{abstract}
Colorectal adenocarcinoma is a major cause of morbidity and mortality. The $\mathrm{Wnt} / \mathrm{ß}$-catenin pathway plays an important role in colon cancers. However, relatively little is known about the regulatory mechanism of $\beta$-catenin in colon cancers. CDK8 is a cyclin-dependent kinase (CDK) member of the mediator complex that couples transcriptional regulators to the basal transcriptional machinery, and is implicated in the transcriptional regulation of key pathways involved in colon cancers. To determine the relationship between CDK8 and $\beta$-catenin expressions, a population-based study was conducted for immunohistochemical staining analysis of tumor tissues, and Western blot analysis and CDK8 interference studies of colon cancer cell lines. The hypothesis that colorectal cancers with CDK8 expression have distinct clinical, prognostic and molecular attributes was tested. Among 127 colorectal cancers, CDK8 expression was detected in $96(76 \%)$ tumors by immunohistochemistry. CDK8 and $B$-catenin expression had significant positive correlation with carcinogenesis, tumor progression and patient survival. Immunohistochemically, CDK8 expression in colorectal cancer was independently associated with $\beta$-catenin activation $(\mathrm{P}=0.0002)$. However, $\beta$-catenin expression was not completely suppressed by CDK8 interference in the colon cancer cell lines HCT-116, HT-29 and SNU-C5. These data support a potential link between CDK8 and B-catenin, and suggest that CDK8 may identify a subset of colon cancer patients with a poor prognosis. However, control of CDK8 is not an effective therapeutic strategy through $\beta$-catenin regulation of general colon cancer.
\end{abstract}

\section{Introduction}

The Wnt/B-catenin pathway involved in the early development process of various cancers is also involved in the development of colorectal cancer $(1,2)$. B-catenin is the central nuclear

Correspondence to: Dr Sung-Chul Lim, Department of Pathology, Chosun University Hospital, 588, Seosuk-dong, Dong-gu, Gwangju, Republic of Korea

E-mail: sclim@chosun.ac.kr

Key words: colon, rectum, adenocarcinoma, CDK8, B-catenin effector in the Wnt signal transduction system. Under physiological conditions, Wnt contributes to the stabilization of B-catenin, and once stabilized, B-catenin is accumulated, and translocates to the nucleus. The impairment of the regulation of the Wnt/ß-catenin pathway (particularly, mutation in APC) plays an important role in the initiation of colorectal cancer (3). Studies on the mechanism of the stabilization of B-catenin within the cytoplams and the translocation to the nucleus has been conducted, nonetheless, the understanding of the mechanism of the accumulation of nonphosphorylated $\beta$-catenin in the nucleus activating the transcription of the target genes cyclin D1, c-myc, c-jun is still insufficient (4).

CDK8 is located in chromosome 13 , forms a pair with transcriptional regulators, and is involved in the regulation of the transcription involved in the formation of colorectal cancer (5). In addition, for $\beta$-catenin-dependent transcription and oncogenesis, activation of CDK8 kinase is essential $(6,7)$.

Therefore, it was determined that studies on CDK8 and B-catenin using human colorectal tissues would provide an important direction for chemotherapy using B-catenin regulators in the future. Therefore, we evaluated the immunohistochemical expression level or pattern of CDK8 and $B$-catenin according to several clinicopathological factors using colorectal cancer tissues, and analyzed the mutual correlation of CDK8 and B-catenin. To confirm the validity of the result of immunohistochemical study perfomed on the colorectal cancer tissues, molecular pathological studies using colorectal cancer cell lines were performed.

\section{Materials and methods}

Case selection and tissue sampling. Among patients who underwent curative surgery for colorectal adenocarcinoma at our hospital from January 1992 to December 2004, non-consecutive 127 patients were selected with relatively well preserved paraffin-embedded tissues, complete medical records, and a known follow-up status. The various clinicopathological parameters of the patients were confirmed by a review of the patient charts and pathology files. Patient survival was confirmed through telephone calls and by mail. Ten cases of colon adenoma and 10 cases of normal-looking colon mucosa, which were all obtained endoscopically, were subjected to immunohistochemical analysis for a comparison study.

Those patients who underwent preoperative chemoradiotherapy and emergency surgery, and those patients that had evidence of hereditary non-polyposis colorectal cancer or 
familial adenomatous polyposis were excluded from the study. Informed consent was obtained from each subject according to the institutional guidelines, and the research protocols were approved by the IRB of our hospital.

\section{Histopathological analysis}

Microscopic examination. Each tumor was re-evaluated by retrospect analysis of the medical records and the tissue slide file of the pathology department, and age, gender, tumor size, histological subtype and the degree of differentiation, the depth of tumor invasion, the status of lymph node metastasis, lymphovascular invasion and distant metastasis, were assessed.

The stage was defined according to the TNM staging system of the American Joint Committee on Cancer (8). Tissues to be examined were fixed in $10 \%$ neutral formalin, and the prepared paraffin-embedded tissues were sectioned 4-5 $\mu \mathrm{m}$ in thickness, and hematoxylin and eosin staining was performed. The cells were examined under a light microscope, the representative area suitable to the study purpose was selected, and slides were prepared for immunohistochemical analysis.

Immunohistochemical staining. Briefly, the $4-\mu \mathrm{m}$ sections that were obtained after formalin fixation and paraffin embedding were deparaffinized in xylene and were rehydrated with distilled water through a graded series of ethanol solutions. The sections were then placed in a glass jar with $10 \mathrm{mM}$ citrate buffer ( $\mathrm{pH}$ 6.0) and irradiated in a microwave oven for $15 \mathrm{~min}$, and were allowed to cool down in the jar at room temperature for $20 \mathrm{~min}$. The slides were then rinsed with Tris buffered saline (TBS). Blocking reagent was added for $10 \mathrm{~min}$ after quenching the endogenous peroxidase activity in $0.3 \%$ hydrogen peroxide for $10 \mathrm{~min}$. The slides were then washed as before, and they were subsequently subjected to the primary antibody reaction. Mouse monoclonal B-catenin (Santa Cruz Biotechnology, Santa Cruz, CA, USA, dilution 1:200) and rabbit polyclonal CDK8 (Santa Cruz Biotechnology, dilution 1:200) applied to the tissue section and they were allowed to incubate in a moist chamber for $1 \mathrm{~h}$ at room temperature. After washing with TBS, Polink-2 HRP plus mouse DAB detection system (Golden Bridge International, Inc., WA, USA) and Polink-2 HRP plus rabbit DAB detection system (Golden Bridge International, Inc.) were used for B-catenin and CDK8, respectively. Counterstaining was performed with Mayer's hematoxylin.

The positive control for CDK8 was mammary invasive ductal carcinoma with strong nuclear staining in another study and for B-catenin was normal colonic mucosa adjacent tumor. Instead of the primary antibody, TBS was used for the negative control.

Analysis and interpretation of the staining. A pathologist who did not know the clinical course of the subjects in order to exclude subjectivity evaluated the staining results. Scoring of $\beta$-catenin was based upon the distribution of $\beta$-catenin within the cell membrane (0-1), cytoplasm (0-2), and nuclei (0-2), with a total score of 0 reflecting cell membrane staining only, similar to that seen in normal colonic mucosa, up to an aggregate score of 5 for tumors with strong nuclear staining (2), diffuse cytoplasmic staining (2), and loss of cell membrane staining (1). Total scores were then collapsed into two grades (inactive, 0-2; active, 3-5) (9).
CDK8 expression was determined by assessing the level of immunohistochemical staining in tumor cell nuclei. Initially, the overall staining intensity was scored as none, weak, moderate or strong. Cases categorized as positive were those characterized by weak, moderate or strong staining, while cases categorized as negative were those with no nuclear staining (10).

\section{Western blot analysis of $C D K 8$ and $\beta$-catenin}

Cell culture. Human-derived colon cancer cell lines HCT116 (11), HT29 (12) and SNU-C5 (13) were seeded with $1.5 \times 10^{6}$ cells in a $10-\mathrm{cm}$ petri dish and maintained in RPMI1640 medium (Invitrogen) containing 10\% FBS (Gibco BRL, Grand Island, NY, USA) and $1 \mathrm{X}$ antibiotic-antimycotic (Gibco $\mathrm{BRL})$ at $37^{\circ} \mathrm{C}$ in $5 \% \mathrm{CO}_{2}$. After overnight incubation, the indicated drugs were treated at various time points. These cell lines were established from colonic adenocarcinoma which had no history of anti-cancer chemotherapy or radiotherapy.

Reagents. Polyclonal rabbit anti-human CDK8 (H-139), monoclonal anti- $\beta$-catenin (E-5) and ECL system were purchased from Santa Cruz Biotechnology and monoclonal mouse anti-actin was purchased from Sigma (St. Louis, MO, USA). Unless specified, drugs were purchased from Gibco BRL.

Western blot analysis. Electrophoretically separated proteins were transferred to an NC membrane, blocked in $5 \%$ skim-milk/TBST, and incubated with primary antibodies to CDK8, $\beta$-catenin and $\beta$-actin. Then, the membranes were incubated with HRP-conjugated secondary antibody and visualized with Super Signal chemiluminescence kit (Pierce Biotechnology), and finally the signals were acquired by image analyzer (Image station $4000 \mathrm{MN}$, Kodak).

RNA interference. siRNA of CDK8 5'-GUU UUU GCC GGU UGU CAA A(dTdT)-3' (S) 5'-UUU GAC AAC CGG CAA AAA C(dTdT)-5' (AS) and scrambled RNA (Ctrl RNAi) sequence 5'-CCU ACG CCA CCA AUU UCG U(dTdT)-3' (S) 5'-ACG AAA UUG GUG GCG UAG G(dTdT)-3' (AS) were designed and synthesized by Bioneer (Daejeon, Korea). Cells $\left(10^{6}\right)$ were transfected with $5 \mu \mathrm{g}$ siRNA using JET-PEI reagent (Polyplus-Transfection, France). Then cells were grown for $48 \mathrm{~h}$ prior to total protein extraction.

Statistical analysis. Stat View software package (Abacus Concepts, Berkeley, CA, USA) was used for statistical analysis. $\chi^{2}$ test, Fisher's exact test, ANOVA, and logistic regression analysis were used to determine the correlation between clinicopathologic parameters and expression patterns of CDK8 and B-catenin. Log-rank test was used for the analysis of Kaplan-Meier survival. Statistical significance was determined at $\mathrm{P}<0.05$.

\section{Results}

Clinicopathological findings. The survival to December 31, 2004 , of 127 cases examined by immunohistochemical analysis in our study was assessed by telephone and mail. The survival record of the longest survival 156 months (average follow-up period: $58.8 \pm 32.7$ months) could be confirmed. One hundred and twenty-seven cases showed $\mathrm{T}$ 
Table I. Frequency of CDK8 expression in colorectal adenocarcinoma according to clinical and pathologic features $(\%)$.

\begin{tabular}{|c|c|c|c|}
\hline $\begin{array}{l}\text { Clinical and } \\
\text { pathologic feature }\end{array}$ & Total no. & $\mathrm{CDK}^{+}$ & P-value \\
\hline All cases & 127 & $96(76)$ & \\
\hline Gender & & & $<0.005$ \\
\hline Male & 66 & $46(70)$ & \\
\hline Female & 61 & $50(82)$ & \\
\hline \multicolumn{4}{|l|}{ T stage } \\
\hline 1 & 5 & $3(60)$ & Referen \\
\hline 2 & 24 & $16(67)$ & \\
\hline 3 & 98 & 77 (79) & $<0.005$ \\
\hline 4 & 0 & 0 & \\
\hline Lymph node & & & $<0.0005$ \\
\hline- & 86 & $58(67)$ & \\
\hline+ & 41 & $38(93)$ & \\
\hline Distant metastasis & & & $<0.0005$ \\
\hline- & 123 & $92(75)$ & \\
\hline+ & 4 & $4(100)$ & \\
\hline \multicolumn{4}{|l|}{ Clinical stage } \\
\hline I & 27 & $19(70)$ & Referen \\
\hline II & 58 & $43(74)$ & \\
\hline III & 38 & $33(87)$ & $<0.01$ \\
\hline IV & 4 & $4(100)$ & $<0.0005$ \\
\hline \multicolumn{4}{|l|}{ ß-catenin, nuclear } \\
\hline- & 53 & $33(62)$ & Referen \\
\hline $1+$ & 40 & $31(78)$ & $<0.05$ \\
\hline $2+$ & 34 & $27(79)$ & $<0.05$ \\
\hline \multicolumn{4}{|l|}{ ß-catenin, cytoplasmic } \\
\hline- & 73 & $46(63)$ & Referen \\
\hline $1+$ & 44 & $33(75)$ & $<0.01$ \\
\hline $2+$ & 10 & $8(80)$ & $<0.005$ \\
\hline ß-catenin, membrane & & & $<0.05$ \\
\hline Expressed & 51 & $32(63)$ & \\
\hline Lost $(1+)$ & 76 & $60(79)$ & \\
\hline ß-catenin, overall score ${ }^{a}$ & & & $<0.0005$ \\
\hline $0-2$ (inactive) & 72 & $43(60)$ & \\
\hline 3-5 (active) & 55 & $45(82)$ & \\
\hline Normal colon mucosa & 10 & $1(10)^{b}$ & \\
\hline Tubular adenoma & 10 & $6(60)^{c}$ & \\
\hline
\end{tabular}

Only significant $\mathrm{p}$-values are described. ${ }^{\mathrm{a}}$-catenin score was calculated as the sum of nuclear $(0-2)$, cytoplasmic $(0-2)$ and membrane (0-1) scores as described in Materials and methods. ${ }^{\mathrm{b}} \mathrm{CDK} 8$ staining was detected as weak $(+)$ positive. ${ }^{\mathrm{c}}$ Tubular adenomas showed 1 weak $(+), 4$ moderate $(+)$ and 1 strong $(+)$ CDK8 staining.

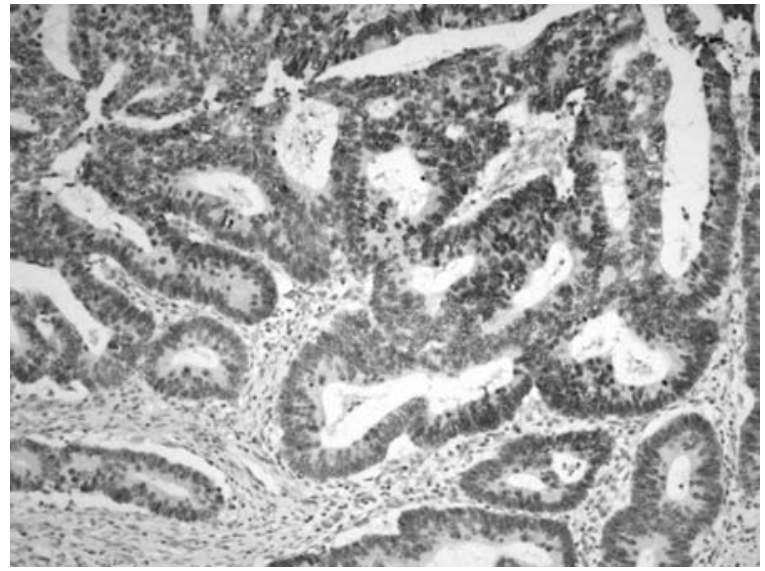

Figure 1. Immunohistochemical staining of colonic adenocarcinoma for CDK8. Tumor cells showed diffuse strong positive nuclear staining. Polink-2 HRP plus rabbit DAB detection system, counterstained by hematoxylin.

disease stage, the presence or absence of regional lymph node metastasis, and the presence or absence of distant metastasis as shown in Table I.

\section{Clinicopathological significance of $C D K 8$}

Immunoreactivity for $C D K 8$. Immunohistochemical staining was performed on 127 cases of colorectal cancer. The disease stage was I-IV. Among them, 96 cases (76\%) showed positive expression of CDK8 in the nucleus (Fig. 1). In the cases that performed the test, examining the rate of the positive expression of CDK8 according to gender, 50 patients of 61 female patients (82\%), and 46 patients of 66 male patients $(70 \%)$ were positive, the females showed a higher positive rate, and it was statistically significant $(\mathrm{P}<0.005)$.

In the normal colon mucosa stained in parallel for comparative observation, 1 case $(10 \%)$ showed positive reaction, and it was weakly positive. In addition, in tubular adenoma cases, 6 cases $(60 \%)$ showed positivity. One case was weak, 4 cases were moderate, and 1 case showed strong positivity (Table I). Such findings were considered to suggest the involvement of CDK8 in the early process of the formation of colorectal cancer.

Interrelation between $C D K 8$ and $T$ stage. The change of CDK8 according to the $\mathrm{T}$ stage was analyzed. In the $\mathrm{T}$ stage 4 patients were not included in the analysis subjects, and thus its interpretation is limited. Nonetheless, as the $\mathrm{T}$ stage became higher, the expression of CDK8 was elevated. The expression of CDK8 was statistically significantly elevated in the T stage III in comparison with stage I $(\mathrm{P}<0.005)$.

Interrelation between CDK8 and lymph node metastasis. Among 41 cases with lymph node metastasis, 38 cases (93\%) expressed CDK8. Among 86 cases without lymph node metastasis, 58 cases (67\%) expressed CDK8. The expression of CDK8 correlated to lymph node metastasis, and it was statistically significant $(\mathrm{P}<0.005)$.

Interrelation between $C D K 8$ and distant metastasis. Only 4 cases with distant metastasis were included in the analysis, and thus it was very limited to interpret statistical significance. Nonetheless, among 123 cases without distant metastasis, 92 cases (75\%) showed CDK8 positivity. Four 


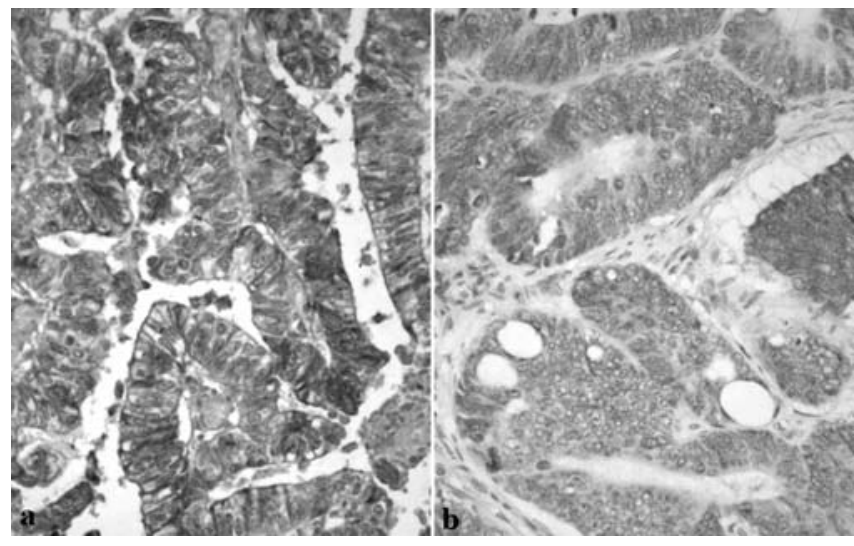

Figure 2. Immunohistochemical staining of colonic adenocarcinoma for ß-catenin. (a) Tumor cells showed diffuse positive nuclear staining and some membranous staining. (b) Tumor cells showed diffuse positive cytoplasmic staining. Polink-2 HRP plus mouse DAB detection system, counterstained by hematoxylin.

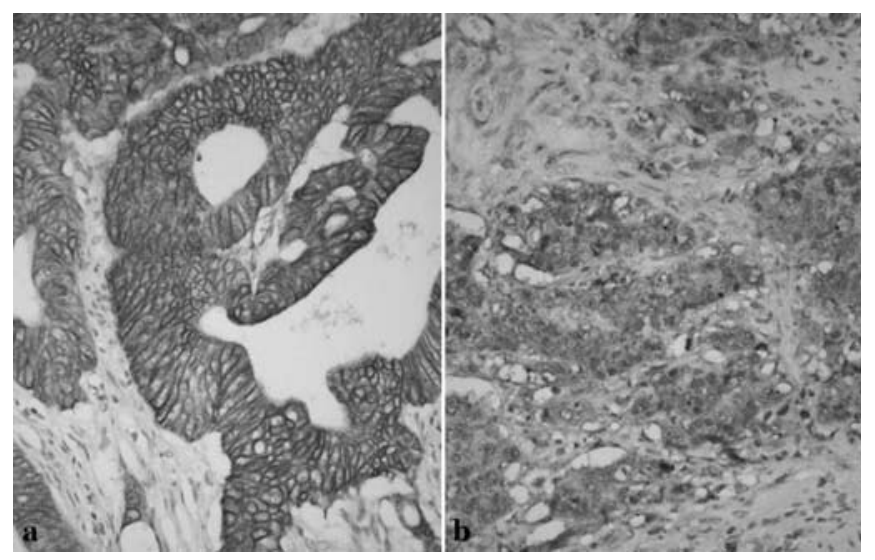

Figure 3. Immunohistochemical staining of colonic adenocarcinoma for B-catenin. (a) Tumor cells showed diffuse positive membranous staining. (b) Tumor cells showed negative membranous staining. Polink-2 HRP plus mouse DAB detection system, counterstained by hematoxylin.

cases with distant metastasis were CDK8 positive, and thus the expression of CDK8 significantly correlated to distant metastasis $(\mathrm{P}<0.0005)$.

Interrelation between CDK8 and clinical stage. There were only 4 patients in clinical stage IV, and thus statistical analysis was limited. The rate of the expression of CDK8 showed a tendency to be increased with the advance of clinical stage, and thus the involvement of CDK8 in the progression of colorectal cancer could be speculated. The rate of the positive expression of CDK8 in the clinical stage III and IV was statistically significantly higher in comparison with the clinical stage $\mathrm{I}(\mathrm{P}<0.01$ and $\mathrm{P}<0.0005$, respectively).

\section{Mutual relation of immunohistochemical expression of} $\beta$-catenin and CDK8

Analysis by intranuclear staining of $\beta$-catenin. Nuclear staining was observed in 74 colorectal cancer cases (58\%). Forty cases $(31 \%)$ showed weak expression, and 34 cases $(27 \%)$ showed moderate/strong expression (Fig. 2). In 53 cases (42\%)

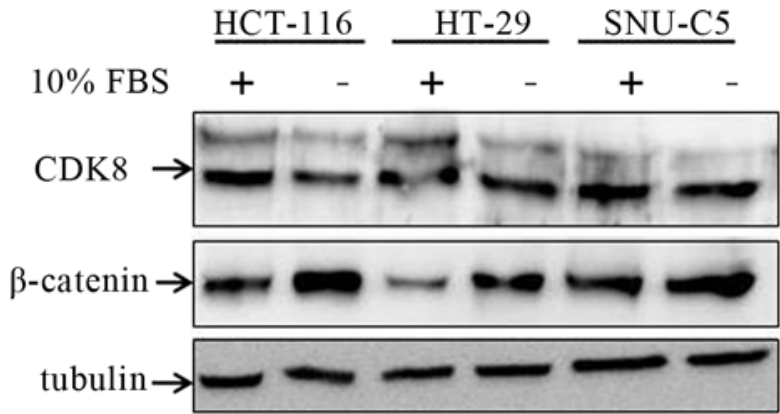

Figure 4. Western blot analysis of colon cancer cell lines for CDK8 and B-catenin. Each cell line expressed CDK8 and B-catenin intensely. After serum starvation for $24 \mathrm{~h}$, CDK 8 level was decreased in each cell line nevertheless, $\beta$-catenin level was increased in each cell line.

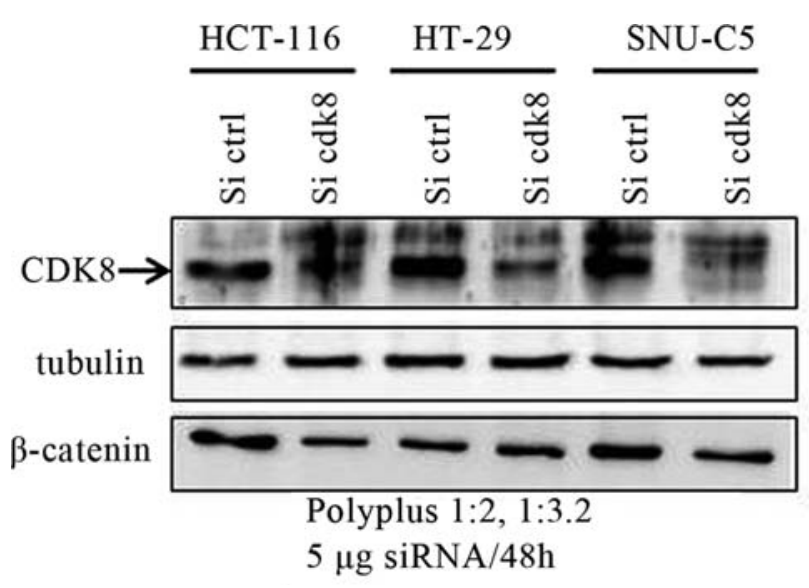

Figure 5. Effect of CDK8 interference on B-catenin expression in colon cancer cell lines. Significant suppression of $B$-catenin was identified in HCT-116 and SNU-C5, but was not significant in HT-29.

there was no expression of $\beta$-catenin, 33 cases $(62 \%)$ showed positive expression of CDK8. In the cases that showed positive expression of $\beta$-catenin within the nucleus, $78-79 \%$ cases showed positive expression of CDK8, and thus the positive expression of $\beta$-catenin in the nucleus correlated to the positive expression of CDK8, and it was statistically significant $(\mathrm{P}<0.05)$.

Analysis by intracytoplasmic staining of $\beta$-catenin. Staining in the cytoplasm was observed in 54 cases of colorectal cancer $(43 \%)$. Forty-four cases $(35 \%)$ showed weak expression, and 10 cases (8\%) showed moderate/strong expression (Fig. 2). Among 73 cases $(57 \%)$ without the expression of $\beta$-catenin within the cytoplasm, 46 cases $(63 \%)$ showed positive expression of CDK8. In the cases that showed positive expression of $\beta$-catenin in the cytoplasm, in regard to the staining intensity, the positive expression of CDK8 was shown in $75 \%$. In cases showing moderate/strong expression, $80 \%$ showed positive expression of CDK8, and thus the expression of $\beta$-catenin within the cytoplasm correlated to the positive expression of $\mathrm{CDK} 8$, and it was statistically significant $(\mathrm{P}<0.01$ and $\mathrm{P}<0.005$, respectively).

Analysis by cytoplasmic membrane staining of $\beta$-catenin. The staining of $\beta$-catenin of cell membrane was lost in 76 cases $(60 \%)$ of colorectal cancer (Fig. 3), and the staining of cell 


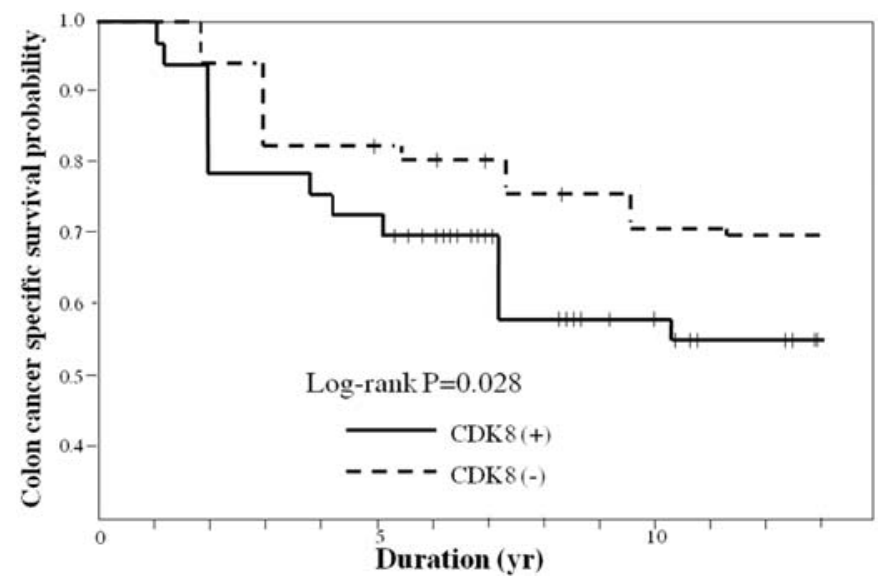

Figure 6. Cumulative survival curves of colon cancer patients by CDK8 expression. CDK8 expression group showed significantly lower survival compared to CDK8 negative group.

membrane was maintained in 51 cases $(40 \%)$. Among the cases that lost the staining of cell membrane, in 60 cases (79\%), the expression of CDK8 was observed, and in the cases maintaining the staining of cell membrane, the expression of CDK8 was observed only in 32 cases $(63 \%)$. Therefore the expression of CDK8 correlated to the loss of the membranous staining of $\beta$-catenin, and it was statistically significant $(\mathrm{P}<0.05)$.

Analysis by $\beta$-catenin total score. In regard to the above mentioned $\beta$-catenin staining, the cases with $\beta$-catenin inactivation corresponding to $0-2$ points of the score summing of the evaluation of the nucleus, the cytoplasm, and cell membrane consisted of 72 of the entire colorectal cases (57\%). The cases with $\beta$-catenin activation that corresponds to 3-5 points consisted of 55 of the entire colorectal cancer cases $(43 \%)$. In the cases with $\beta$-catenin activation, the rate of the expression of CDK 8 was $82 \%$, on the other hand, in the cases with $\beta$-catenin inactivation, the rate of the expression of CDK8 was $60 \%$, the expression of CDK8 correlated to $ß$-catenin activity, and it was statistically significant $(\mathrm{P}<0.0005)$.

\section{Colon cancer cell line study}

Western blot analysis - expression of CDK8. Colon cancer cell lines were cultured, and CDK8 was measured. It was strongly expressed in HCT-116, HT-29 and SNU-C5 cell lines (Fig. 4). This suggests the involvement of CDK8 in colorectal cancer as shown in the results of immunohistochemical staining.

Expression of $\beta$-catenin. Colon cancer cell lines were cultured and the expression of $\beta$-catenin was assessed. It was shown to be expressed strongly in HCT-116, HT-29 and SNU-C5 cell lines. However, in HT-29 cases, the expression of B-catenin was slightly weaker than HCT-116 and SNU-C5 (Fig. 4). This suggests that $\beta$-catenin could be expressed by the involvement of CDK8 in colorectal cancer as shown in the result of immunohistochemical staining.

Experiments in the absence of serum. To elucidate the relationship of CDK8 and $\beta$-catenin, colorectal cancer cell lines were cultured for $24 \mathrm{~h}$ in serum-free medium. In the absence of serum factors, the expression of CDK8 was reduced slightly in HCT-116, HT-29 and SNU-C5 cell lines. However, $\beta$-catenin showed a tendency to be expressed more strongly in the medium without serum factors (Fig. 4). In other words, under the condition where serum factors were omitted and thus CDK8 was reduced, $\beta$-catenin showed a tendency to be increased.

Expression of $\beta$-catenin after treatment of CDK8 siRNA. CDK8 interference was generated, and the expression of B-catenin in the colon cancer cell lines, HCT-116, HT-29 and SNU-C5, was assessed. The expression of $\beta$-catenin was decreased noticeably in HCT-116 and SNU-C5; nonetheless, it could not be suppressed completely. In addition, in HT-29 cases, reduction of the expression of $\beta$-catenin was not observed (Fig. 5).

Survival analysis by CDK8 expression. To analyze the outcome of patients according to the expression of CDK8, the effect of CDK8 on the survival of colorectal cancer patients was analyzed. The overall 5-year survival rate of patients was $73.3 \%$. At the time of examination, among 127 patients (average follow-up period: 4.9 years), 75 patients survived, and $59.1 \%$ survival rate was shown. Among the CDK8 negative group (31 patients), 22 patients $(71 \%)$ survived. In the CDK8 positive group (96 patients), 53 patients $(55 \%)$ survived (log-rank: $\mathrm{P}=0.028$ ) (Fig. 6). Hence, it was found that the expression of CDK8 was closely associated with the high mortality of colorectal cancer patients.

\section{Discussion}

The WNT gene as well as its product comprise the WNT signal pathway, and the molecule playing a central role in this signaling pathway is $\beta$-catenin, and its stability is regulated by the APC complex. When WNT receptor is inactivated, $B$-catenin is localized in the membrane protein E-cadherin ( $C D H 1)$, and kinases in the APC complex phosphorylate $\beta$-catenin in the cytoplasm resulting in the induction of its rapid degradation. On the other hand, when WNT is activated, kinases in the APC complex are suppressed resulting in $B$-catenin accumulating in the cytoplasm, translocating to the nucleus, and stimulating the transcription of various target genes (14). In addition, in the WNT pathway, the TGF- 3 pathway (15) and the COX-2/ prostaglandin pathway are involved in cross-talk (16-18).

The activation of WNT/ß-catenin signal has been reported to be involved in the development of various human cancers including colorectal cancer; nonetheless, studies on the relationship of CDK8 and $ß$-catenin in colorectal cancer are meager (19-21).

To characterize the molecule in charge of the role of posttranslational modifier after the translation of ß-catenin, Firestein et al (6) conducted high-throughput screening studies and found that only CDK8 resided in a region of copy number gain in nearly half of colon cancers. In addition, they confirmed by murine cell culture studies in the cases expressing CDK8, but not a kinase inactive version of CDK8, transformed immortal murine cells. Based on such a background, it was elucidated that CDK8 is an oncogene that 
is amplified in colorectal cancer. Most CDKs play an important role in the regulation of cell cycle. CDK7, CDK8 and CDK9 regulate gene expression by the interaction with the transcriptional machinery. Particularly, together with its partners cyclin C, MED12 and MED13, CDK8 forms a large protein mediator complex 'CDK8 module' that plays a central role in the regulation of transcription. In addition, the components of the CDK8 module MED12 and MED13 act as the regulator of $\beta$-catenin activation $(3,22,23)$. In addition, CDK8 could increase $\beta$-catenin by an indirect method, which is a method through E2F1 that suppresses B-catenin. Thus, CDK8 suppresses E2F1, and at the end, the ability of E2F1 suppressing $\beta$-catenin is reduced resulting in the increase of $\beta$ catenin (7). In addition, CDK8 is involved in the regulation of the cell cycle of normal cells by binding of the basal transcriptional machinery to transcription factors such as Notch (24) or p53 (25).

To confirm the relationship of CDK8 and B-catenin based on colorectal cancer tissues, immunnohistochemical studies were performed. Based on CDK8 staining performed on the normal colon mucosa, tubular adenoma and adenocarcinoma, it was speculated that CDK8 is involved not only in the early stage of the formation of colorectal cancer but also in the progression and metastasis of adenocarcinoma. In addition, CDK8 was significantly associated with the expression pattern of $\beta$-catenin, it positively correlated to the expression of $\beta$ catenin in the nucleus or in the cytoplasm and inversely correlated to the staining of $\beta$-catenin on cell membrane, and thus the possibility whether WNT/ß-catenin signal pathway could be controlled through CDK8 became of interest. It is thought that the activation of $\beta$-catenin through the regulation of CDK8 may widen the understanding of the development of colorectal cancer and also the understanding of the progression of colorectal cancer.

The experiments using colorectal cancer cell lines performed to assess the relationship of CDK8 and $\beta$-catenin comprehensively showed the results slightly lower than expectation, and thus it is considered that the relation of the regulation of $\beta$-catenin by CDK8 assessed through immunohistochemical staining may require slightly different interpretation. CDK8 infeference was induced in the colon cancer cell lines expressing $\beta$-catenin strongly by the application of siRNA, and the alteration of the expression of $\beta$-catenin was examined. Noticeable reduction was observed in some cases; nonetheless, complete suppression was not shown, and in some cases, the expression of $\beta$-catenin was only slightly reduced, and thus it is considered to imply that in the expression of $\beta$-catenin, factors other than CDK8 are also involved. In addition, in the Western blot analysis performed in the absence of serum factors, CDK8 was reduced but the expression of $\beta$-catenin was rather elevated, which renders the hypothesis that the expression of $\beta$-catenin is influenced by CDK8 primarily doubtful. It is thought that such results are contradictory to the proposal of Firestein et al (6) that only CDK8 plays a role of post-translational modifier of $\beta$ catenin.

Firestein et al (6) produced the colon cancer cell line DLD1 that has the APC deletion and proliferate in the B-catenindependent manner, performed the supplement experiments using the colon cancer cell line HCT-117 with ß-catenin- dependent proliferation, and reported the study results. The colon cancer cell lines used in our study were HCT-116, HT-29 and SNU-C5, and it is considered that the characteristics of these cells are different from DLD1. Based on their results, Firestein et al (6) reported that CDK8 plays an important role in the expression of $\beta$-catenin, and based on this, they strongly suggested the possibility of treatment of colon cancer through the regulation of CDK8. It is considered that it is reasonable to revise the theory as it is limited to a specific colon cancer.

In addition, it is thought that to accept directly the result of in vitro experiments as the theory of the development and progression of general tumors without additional validation procedures may be very limited. Nevertheless, based on our study performed on tumor tissues, it was confirmed that CDK 8 and $B$-catenin play a very important role in the development, progression and metastasis process of colon cancer. In addition, it could be confirmed that the effective regulation of the expression of $\beta$-catenin through CDK8 is the proposal applicable only to some limited cases of colon cancer, and not applicable to all colon cancer. Therefore, it was understood that also the theory concerning the development of the treatment method for colon cancer through the regulation of CDK8 is applicable to certain very limited colon cancer.

\section{Acknowledgements}

This study was supported by National Research Foundation of Korea (NRF) Grant funded by the Ministry of Education, Science and Technology (MEST) through the Research Center for Resistant Cells (R13-2003-009).

\section{References}

1. Walther A, Johnstone E, Swanton C, Midgley R, Tomlinson I and Kerr D: Genetic prognostic and predictive markers in colorectal cancer. Nat Rev Cancer 9: 489-499, 2009.

2. Powell SM, Zilz N, Beazer-Barclay Y, Bryan TM, Hamilton SR, Thibodeau SN, Vogelstein B and Kinzler KW: APC mutations occur early during colorectal tumorigenesis. Nature 359: 235-237, 1992.

3. Firestein R and Hahn WC: Revving the throttle on an oncogene: CDK8 takes the driver seat. Cancer Res 15: 7899-7901, 2009.

4. Oving IM and Clevers HC: Molecular causes of colon cancer. Eur J Clin Invest 32: 448-457, 2002.

5. Kim S, Xu X, Hecht A and Boyer TG: Mediator is a transducer of Wnt/beta-catenin signaling. J Biol Chem 281: 14066-14075, 2006.

6. Firestein R, Bass AJ, Kim SY, Dunn IF, Silver SJ, Guney I, Freed E, Ligon AH, Vena N, Ogino S, Chheda MG, Tamayo P, Finn S, Shrestha Y, Boehm JS, Jain S, Bojarski E, Mermel C, Barretina J, Chan JA, Baselga J, Tabernero J, Root DE, Fuchs CS, Loda M, Shivdasani RA, Meyerson M and Hahn WC: CDK8 is a colorectal cancer oncogene that regulates beta-catenin activity. Nature 455: 547-551, 2008.

7. Morris EJ, Ji JY, Yang F, Di Stefano L, Herr A, Moon NS, Kwon EJ, Haigis KM, Naar AM and Dyson NJ: E2F1 represses beta-catenin transcription and is antagonized by both $\mathrm{pRB}$ and CDK8. Nature 455: 552-556, 2008.

8. Greene FL, Page DL and Fleming ID: AJCC Cancer Staging Manual, 6th edition. Springer-Verlag, New York, 2002.

9. Kawasaki T, Nosho K, Ohnishi M, Suemoto Y, Kirkner GJ, Dehari R, Meyerhardt JA, Fuchs CS and Ogino S: Correlation of beta-catenin localization with cyclooxygenase-2 expression and $\mathrm{CpG}$ island methylator phenotype (CIMP) in colorectal cancer. Neoplasia 9: 569-577, 2007. 
10. Firestein R, Shima K, Nosho K, Irahara N, Baba Y, Bojarski E, Giovannucci EL, Fuchs CS and Ogino S: CDK8 expression in 470 colorectal cancers in relation to beta-catenin activation, other molecular alterations and patient survival. Int J Cancer 126: 2863-2873, 2010

11. Brattain MG, Fine WD, Khaled FM, Thompson J and Brattain DE: Heterogeneity of malignant cells from a human colonic carcinoma. Cancer Res 41: 1751-1756, 1981.

12. Fogh J (ed): Human Tumor Cells in vitro. Plenum Press, New York, pp115-159, 1975

13. Park JG, Oie HK, Sugarbaker PH, Henslee JG, Chen TR, Johnson BE and Gazdar A: Characteristics of cell lines established from human colorectal carcinoma. Cancer Res 15: 6710-6718, 1987.

14. Clevers H: Wnt breakers in colon cancer. Cancer Cell 5: 5-6, 2004.

15. Attisano L and Labbe E: TGFbeta and Wnt pathway cross-talk. Cancer Metastasis Rev 23: 53-61, 2004.

16. Buchanan FG and DuBois RN: Connecting COX-2 and Wnt in cancer. Cancer Cell 9: 6-8, 2006

17. Castellone MD, Teramoto H, Williams BO, Druey KM and Gutkind JS: Prostaglandin E2 promotes colon cancer cell growth through a Gs-axin-beta-catenin signaling axis. Science 310: 1504-1510, 2005.

18. Araki Y, Okamura S, Hussain SP, Nagashima M, He P, Shiseki M, Miura K and Harris CC: Regulation of cyclooxygenase-2 expression by the Wnt and ras pathways. Cancer Res 63: 728-734, 2003
19. Samowitz WS, Slattery ML, Sweeney C, Herrick J, Wolff RK and Albertsen H: APC mutations and other genetic and epigenetic changes in colon cancer. Mol Cancer Res 5: 165-170, 2007.

20. Zeng G, Germinaro M, Micsenyi A, Monga NK, Bell A, Sood A Malhotra V, Sood N, Midda V and Monga DK: Aberrant Wnt/ beta-catenin signaling in pancreatic adenocarcinoma. Neoplasia 8: 279-289, 2006

21. Thorstensen L, Lind GE, Lovig T, Diep CB, Meling GI, Rognum TO and Lothe RA: Genetic and epigenetic changes of components affecting the WNT pathway in colorectal carcinomas stratified by microsatellite instability. Neoplasia 7: 99-108, 2005.

22. Casamassimi A and Napoli C: Mediator complexes and eukaryotic transcription regulation: an overview. Biochimie 89: 1439-1946, 2007

23. Gold MO and Rice AP: Targeting of CDK8 to a promoterproximal RNA element demonstrates catalysis dependent activation of gene expression. Nucleic Acids Res 26: 3784-3788, 1998.

24. Fryer CJ, White JB and Jones KA: Mastermind recruits CycC:CDK8 to phosphorylate the Notch ICD and coordinate activation with turnover. Mol Cell 16: 509-520, 2004.

25. Donner AJ, Szostek S, Hoover JM and Espinosa JM: CDK8 is a stimulus-specific positive coregulator of p53 target genes. Mol Cell 27: 121-133, 2007. 